\title{
Noosphere as Optimal Control. Part 2. Reflective noosphere ${ }^{1}$
}

\author{
Boris Balter
}

\author{
Researcher, Russian Space Research Institute (Moscow, Russia) \\ E-mail: Balter@mail.ru \\ ORCID: 0000-0003-4346-8052
}

\author{
Marina Faminskaya \\ PhD, Associate Professor, Russian State Social University (Moscow, Russia) \\ E-mail: Faminskaya@mail.ru \\ ORCID: 0000-0001-9044-4382
}

\begin{abstract}
Conceptual system developed in optimal control theory for technical purposes is used as a philosophical instrument applied to cyclic information processes, which are expected to be the basis of noosphere. Noosphere was perceived by the founding fathers of this concept, Vernadsky, Teilhard de Chardin, e.a. as an outgrowth of the evolutionary process, which begins with cosmogenesis and proceeds through geosphere and biosphere. We attempt to apply the optimal control concepts to all three levels - geospheric, biospheric, and noospheric - due to their having a common structure of information processes (or entropic processes considered as proto-information). These processes include homeostasis, accumulation and expenditure of information, formation of hierarchical information structures, evolution involving the breaks of homeostasis etc. In noosphere, controlled system may have the same informational capabilities as controlling system, so that the term "dialog" is more adequate; in this case, we extend optimal control description to game theory. The cyclic, feedback logic of optimal control seems better adapted to noospheric processes than usual causeeffect logic.
\end{abstract}

This second part of the paper proceeds from the geo- and biospheric levels discussed in the first part to the noospheric level. The basic structure at this level is the fusion of natural matter/energy cycles characteristic for geosphere with anthropogenic information cycles, which extend information accumulation and adaptation inherited from biospheric level into reflective realm. The basic type of informational interaction between these structures is construed in perspective of game theory between reflective players. Its essential feature is the interaction between reflective images that each player forms of other players and of oneself. We describe the nontrivial information flows that can arise in a distributed global system of such structures, including complex interactions between collective and individual levels and paradoxes. We analyze the role of science as the carrier of the "rational model", on which this entire system is based, and the impact on this model of the sociobiological background of science inherited from the biospheric level. We also discuss the role of natural language as an alternative noospheric structure capable of supporting the irrational components of the noosphere. City is discussed as an example of an emerging structure integrating

\footnotetext{
${ }^{1}$ The work was supported by the Russian Ministry of Education and Science (task1.9328.2017).

(C) Balter, Boris, 2018

(C) Faminskaya, Marina, 2018
} 
the basic noospheric components, albeit in an inchoate form. Finally, we consider the reflective identities ("I"), which may emerge in noosphere, and the relevant ethical issues.

Keywords: optimal control, game theory, reflection, noosphere, information cycles, diachronic, paradoxes.

Received 25 May 2017; accepted 29 June 2017

Philosophy and Cosmology, Volume 20, 2018: 56-73

DOI: $10.29202 /$ phil-cosm/20/5

\section{Noosphere: network of reflective games with nature}

Here, we follow part 1 of this paper [Balter \& Faminskaya, 2017] up the GBN hierarchy (geo-, bio-, noospheric ladder) to noosphere. The enumeration of chapters continues from [Balter \& Faminskaya, 2017].

\section{1 "Control" of learning systems as a game}

Extension of $O C$ to equal partners. In this paragraph, we consider the next level of optimal control (OC) complexity where both object and subject are capable of information processing and learning (reflective systems come further on). The difference between subject and object of OC becomes relative: a system may be considered as subject or object, according to the reference frame chosen. Graphically, the change of reference frame, which swaps subject and object, amounts to a half-turn of OC cycle and produces a covariant change of OC equations swapping the object's variables and the subject's variables (see Chapter 1 of [Balter \& Faminskaya, 2017]). As a result of this covariance, we call both partners. Each partner has its own criterion to be optimized. Neither informational capabilities nor controls and observables need be identical for partners. E.g., the former object is expected to be able to observe the former subject only by perceiving its control efforts and to control it only by demonstrating to it some observables, which are deliberately rigged up. Each partner can actively sound the other by generating the sounding information, which returns to it after tracking a full OC cycle. However, each partner's sounding information is defined with respect to this partner's criterion as to a reference frame, so that it may be perceived by the other as noise or may be misinterpreted in the other's reference frame. These two types of information (or more if there are more partners) are fused in the signal flow in OC, and their deconvolution is known to be possible only if certain prior information (alias, reference frame) is available.

As both partners now have information structures (models), their description in OC formalism, including the models of each other's state, is more complicated and hierarchical. We will simplify the picture by assuming that there is no direct exchange of higher-level information between partners because their models, generally, use different "languages". Thus, the horizontal information cycle runs only at the lowest informational level - that of actions, which are assumed to be universally understandable and serve as signals. Further, we will discuss changes to this picture introduced by direct informational exchange at higher levels.

Transition from OC to GT. Partners with their own optimized criteria are a subject of game theory (GT) rather than of OC. Transition from OC to GT can be quite smooth. E.g., consider the original OC homeostasis where the subject "helps" the object to damp variations of environment. Then, there is a single common criterion optimized by both the object and 
the subject. If later on the object acquires a degree of independence and forms its own goal and criterion (or if, equivalently, the subject's goal deviates from keeping the original homeostasis), this criterion will split in two. In such a continuous transition from OC to GT, the symptoms of object's "getting loose" can be recognized by the subject as the increase of information flow from object, which will now surpass the information embedded by subject into object in the process of control.

Prisoners' Dilemma (PD) is a salient example of GT, which captures the essential features of games. In a single-round PD, two partners have to decide (simultaneously and without exchanging any information) whether to cooperate or defect. Mutual cooperation is most favorable for both, mutual defection is moderately unfavorable, but the situation most unfavorable for a partner happens if it cooperates and the other partner defects. How can a partner avoid such a situation? Only by a priori defecting, so that both will do so and miss their potential cooperative gain. This is an archetypal example of contradiction between collective and individual criterion. It can be solved if both partners harbor an additional level of information: collective values, which produce mutual trust. In a singleround $\mathrm{PD}$, this information can come only externally, e.g., from culture. In a multi-round PD, this information can accumulate in the same way it accumulates in OC (trust buildup). There were several tournaments where computer programs interacted in such iterated PDs [Axelrod, 1997]. Each program used its own algorithm (strategy, in GT terms) for gathering information about partners and for deciding from this information whether to cooperate or not on encountering a familiar or unfamiliar partner. The tournaments were later extended with simulated "natural selection" of programs based on their success in such encounters and mimicking the biospheric evolution. It turned out that programs with a balanced approach to cooperation and competition were, generally, more evolutionarily stable. Another advantageous feature was the "transparency" of program's strategy, which makes eliciting the strategy from program's behavior not too difficult for its partners. Thus, ease of reflection can be important.

Homeostasis and information exchange in games. In GT, the notion corresponding to homeostasis is equilibrium. In Nash equilibrium, the unilateral deviations from it by any partner would conflict with the individual criterion of this partner (taking into account the reaction by others). In Pareto equilibrium, multilateral actions are considered, homeostasis is usually a domain, not a single state, and partners can exchange information and bargain in search of a better place within it (e.g., in PD, their Pareto equilibrium is mutual cooperation, and they would find it if they were able to communicate). Even if partners' criteria place on them conflicting demands, the dialog between partners may be essential for preserving a homeostasis unreachable for any partner individually. E.g., homeostasis of noosphere should involve interaction between ecological, societal, technological and other functional systems as "players" in the modern world. The vital dynamical character of this "game" between economic and technological systems is very clear nowadays. It is an essential feature of current homeostasis due to immensely increased information flows. This feature is captured by the concept of sustainable development, which sees the emerging noosphere as a bicycle: to keep its equilibrium, it has to roll on.

Interaction between partners can be seen in evolutionary perspective: as mutual catalysis, which helps to emerge from unfavorable local extrema. Nash equilibrium in PD (mutual defecting) is such an extremum, and information exchange between partners, e.g., by repeated interaction, leads to cooperative emergence from it. In the noosphere, hopefully, cooperative actions will emerge not only from evident mutual benefit but also in less evident situations, 
due to intensive information exchange between individuals and between individual and collective values, as in PD example. However, within OC or GT framework, cooperation cannot be viewed as morally better by definition. The only argument for it is increased gain, and the same argument may favor competition when it is more lucrative (individually or collectively). This is a limitation of OC and GT since even nowadays - and even more so, in the noosphere - human decisions are not as simply motivated as that.

Reflection accompanying information exchange. We considered, so far, information exchange that takes place only through actions and implies constructing models of partners only from their observed actions. Such models naturally tend to develop a reflective structure (see Chapter 1 of [Balter \& Faminskaya, 2017]). They have an inherent uncertainty: the partners' actions can be interpreted correctly or incorrectly. If a partner misinterprets the other's actions, its own actions will be misguided and, thus, prone to misinterpretation by the other partner, so that a vicious circle of accumulating misunderstanding will develop. Alternatively, each partner can catalyze the accumulation or correct information in other partners. In this case, virtual information generated by a partner first as a scenario of actions based only on phantom images of other partners will become actual information when reinforced by partners' actions. This is like actualization of virtual genetic information described in Chapter 3 of [Balter \& Faminskaya, 2017], with the role of “environment" being played by other partners.

Even if there is a beneficial cooperation between partners, it does not necessarily drive them to reflection about each other or about the motives of their cooperation. However, when such reflection emerges, it has a distinct moral relevance. Neither cooperation per se (observed even in animals), nor reflection per se (observed in warring humans) is moral, but the reflected cooperation is. In noosphere, the interaction with natural environment should acquire a cooperative and reflective character and would become one of the major moral issues (see more on it below).

\subsection{Hierarchy of reflections. Paradoxes of reflection}

Reflection as an outgrowth of geo- and biosphere. We will consider here only "natural" reflective games as a continuation of evolving geo- and biospheric OC. This excludes as "unnatural" the majority of ingenious reflective games invented by humans. In what remains, the notable difficulty of describing reflection is alleviated by the information flow approach. In particular, the formation of high reflective levels is seen as evolution of hierarchical information structures, which is not without a cost, both informational and material. Thus, the tendency to form reflection is seen as a continuation of the tendency of adaptive control to form multilayer models and of the general evolutionary tendency to form higher peaks of complexity.

In this perspective, human reflection is not just a profitable biological "investment" rather, it is a reflective organ of entire noosphere. Reflection already begins to permeate the emerging noosphere, and through it, the bio- and geosphere. Therefore, we can expect the dissolution of differences between natural and artificial OC cycles, so that "control of nature" will look more like a "reflective game with nature". Smart gadgets, which predict human actions, are a prototype of potential partners in this game. However, this does not mean that all reflective systems are on a par: not all reflective levels are made equal, the information inhabiting them is qualitatively different, and supporting the information flow from one level to another is a theoretical and practical problem.

Reflection as generator of paradoxes. If some reflective level is conventionally designated as "reality" (including the zero level, which is reality proper), its image at the next reflective 
level can be tagged "symbol". A two-way mapping between these levels is an information flow, which can be trivial or nontrivial, and, in particular, paradoxical. E.g., the meaning of a textual description of reality usually is without problems projected back onto reality defining our actions in it. However, when a text speaks reflectively about itself, the mapping can be a paradox (e.g., the Epimenides paradox [Hofstadter, 1999]). An eminent archetype of this kind of paradox is the incompleteness theorem by Gödel. There, going from a logical formula to its translation into a number is what we call mapping up the reflective hierarchy. Conversely, translating the number's factorization into the proof of the logical formula is what we call mapping down the reflective hierarchy, and these two mappings are incompatible.

Considering Epimenides-like information structure as a partner in dialog with humans, we see that it contains a kind of intrinsic "logical flow", which is revealed in the corresponding flow it induces in human mind: ceaseless switching between "it is true" and "it is false". In Gödel's context, the resolution of this uncertainty lies in extending the reflective informational structure by adding some external information, to settle the uncertainty either to "true" or to "false". Of course, the extended system may contain its own paradoxes. Thus, paradoxical relationship between reflective levels can generate information: set up questions that attract information from the environment. Reflective information flows run in "vertical" reflection time as a part of optimization of the next step in "horizontal" real time. If reflective processes are too complicated or do not converge, as in the case of a paradox, they may spill out into "horizontal time" as delays in decision-making.

Relationships between images at different reflective levels. In Chapter 1 of [Balter \& Faminskaya, 2017], the term "phantom" was used for representation of a partner within reflective informational levels of another partner. Here, we use the term "image" because "phantoms" can become almost real: interaction between them influences the real behavior of partners within whom they reside. Consider the simplest case: a one-step game where real partners reach a Nash equilibrium assuming that all others act as is best for them. Non-reflective approach postulates that each partner has $100 \%$ accurate images of others' criteria and possible actions. If not, the game becomes reflective. Each partner optimizes its own actions not against real partners but against the images of them, which, in turn, may contain their images of this partner, etc., ad inf. Equilibrium may or may not exist in this situation, depending on reflective information structures within each partner. Even if first-level images accurately reflect the real partners' state, it is no guarantee that the images of partners' higher levels are as accurate.

Usually, to preserve continuity with Nash equilibrium, it is assumed that criteria optimized are those of real partners only (e.g., in [Novikov \& Chkhartishvili, 2014]). Then, each partner, although having a complicated multilayer information structure, acts as an integrated "I." In reflective calculation of other partners' actions, the principle "assume they do as is best for them" is applied to these partners' images, not to real players, but this does not mean that images become in any way independent players. However, this assumption is a simplification of what happens in real life. E.g., optimizing the opinion of others about oneself may become one's main criteria of behavior. In OC language, this means restructuring of the real-level criterion under the influence of information flow coming from higher reflective levels of model hierarchy. This can take place in a collective, as well as within an individual. Ideologies are especially apt in projecting collective images from collective reflective structures into individuals, so that what one optimizes is one's image allegedly formed by another (collective) image. This is an example of second-level reflection. Such processes become especially effective when the images do not just catalyze the real processes, as any informational structure is bound to do, but auto-catalyze themselves as memes (see below). 
As a typical result of such self-amplification, an ancillary part of the criterion comes to dominate the criterion and the means become the goals.

Reflection forms virtual reality. Thus, there is a complex interaction between reflective levels, which can be metaphorically described, in contrast to "reflective game", as a "game of reflections". As a result, there is uncertainty of what reflective level to use as a portrait of the real situation and as a basis for action: "I don't know what I really think about that". When different images of a situation multiply in a single player, the difference between real and virtual situation blurs. The unconscious tag, which our psyche places on inner images and which helps distinguish reality from a plausible dream, gets more or less arbitrarily shifted along the hierarchy of reflective levels. Some level is selected as a basis for action, but it is accompanied by a suite of virtual alternatives, which can capture the tag "reality" anytime they are better supported (e.g., by observation). The blurring of the divide between real and virtual, called "virtual reality", comes from hyper-activation of the vertical information transfer between reflection levels. This is expected to be a prominent feature of noosphere. However, reflection comes at a cost, which increases with the level of reflection, so that very high levels are inaccessible to human brain. Reflection cost is an instance of matter/ energy cost that any information flow requires. Computer games producing virtual reality are effective because they minimize this cost.

Reflection and collectives. A collective can participate in reflective games in two roles: as a peer-level partner or as a center, which applies reflective control to individuals. Reflective control, rather than affecting the actual state of individual, is targeted at reflective images of oneself or of others. This is done by presenting the appropriate information to individual either directly shaping the reflective images or doing it indirectly, through real facts and events selected to support the necessary reflective images within the individual. A collective information cost has to be incurred, but the material gain to the collective from the imposed individual actions predominates in the collectively optimized criterion.

To apply reflective control, a collective needs not necessarily have a full model of the reflective structure of individuals. This is unlikely because reflective capabilities of an average collective are much lower than of an average individual. Rather, to perform reflective control, a collective uses the reflective capabilities of its component individuals "instinctively", as a person uses one's body members. Although the biospheric natural selection is targeted at collectives, its edge directed toward noosphere works with individuals due to their reflective capabilities. Thus, tensions between individual and collective existing in biosphere are inherited and amplified in the noosphere. Consider the problem of authority/power/rule, which is based on self-identification of an individual with the collective like ' $L$ 'etat, c'est moi". Another example is xenophobia and war based on the replacement of a full reflective image of partners by the primitive dichotomy "us - them". Such a simplification of complex interlaced reflections between "them" and "us" saves the resources by canceling reflective information flows and thus helps to optimize the criterion. Yet another example is the gap between high information processing capabilities of humans and primitive "linear logic" used in reflection about others - a product of primitive transactions between individuals, e.g., in trading, so that it can be called "market logic". Ironically, the actual logic by which market functions (optimization called "invisible hand") is so complex that modern economics is still far from fully deciphering it.

Reflective images in noospheric control. Just as genetic "images" of environment are of a different nature than real environment (see Chapter 3 of [Balter \& Faminskaya, 2017]), the reflective images of reality are of a virtual rather than real nature. Genetic "images" are 
projected (incarnated) into phenetic features. Noospheric reflective images are projected into reality through conscious control based on them. Due to reflective character of prototype images, their incarnations always have, in addition to the practical, functional side, a symbolic side. This is specific to noosphere and absent in geo- and biosphere. Some of these images have a specific autocatalytic nature, by means of which they amplify and multiply themselves when getting into a favorable reflective environment, individual or collective. These are the noospheric analogs of viruses called memes in [Dawkins, 2006]. Viruses are currently understood as a necessary element of evolution at its primitive, prokaryotic stage because they provide a fast dissemination of genetic improvements by means of horizontal gene transfer. Analogously, memes perform "horizontal transfer" of information across reflective model layers between individuals or collectives, short-circuiting the "big" information cycle, which downloads information to the substrate real OC embedded into environment where information is checked against observed reality. Memes are quasi-independent of reality.

In contrast to memes, the virtual images produced by reflection, although having no exact counterpart in reality, still enrich the model layer because after passing the "big" information cycle, they bring back to reflective layers the information on their discrepancies with reality, which serves to correct reflection. In addition, when used in active sounding (see Chapter 1 of [Balter \& Faminskaya, 2017]), they may "resonate" with the corresponding reflective images in the partner, e.g., in developing mutual trust or distrust as described above. Reflective images preserve this potential even outside a "live" dialog, e.g., when materialized as texts. Obviously, such materialized reflective structures as a part of culture are an essential and the most conspicuous part of noosphere. They are collective information structures that play the role of a catalyzer stimulating and directing the dialog between individuals: between the author and the reader and between different readers. This dialog is diachronic, as are many things connected to noosphere. Cultural diachrony is a by-product of bidirectionality of OC time (Chapter 1 of [Balter \& Faminskaya, 2017]). As [Mandelstam, 2012] puts it: "Dante is a tool of folding and unfolding time."

Reflection about the noosphere. This text, like everything written and to be written about the noosphere, is a reflective informational structure of this same noosphere. Thus, it is reflection about reflection - meta-reflection, which may be a new type of reflection, unique for the noosphere. This specificity of noospheric information structures applies to scientific texts, as well, so that they have to overcome the traditional posture of natural science as an activity more reflective than its objects (see 4.4).

\subsection{Noospheric models: informational catalyzers of material flows}

Distributed reflective control in noosphere. Noospheric "controllers" (alias, partners in dialogs), being primarily information structures, are expected to actively exchange information, thus forming distributed "control" systems. The nodes of such systems can represent different spatial locations, different components of the systems' states or different functional subsystems, e.g., different natural cycles. These noospheric collectives will inherit much from the laws of organization of biospheric collectives, due to common OC laws. They will also inherit from "computational ecosystems" [Huberman, 2001]. Distributed control was considered in Chapter 1 of [Balter \& Faminskaya, 2017] assuming a gap between informational capabilities of the subject and object of control. In noosphere, reflective relationship between subjects and "smart objects" of OC is expected to make each distributed OC node a fusion on informational and material cycles, in which the distinction between anthropogenic and natural cycles would get blurred. Therefore, our usage of the 
terms "subject" and "object" below is just a convention meant for preserving the continuity with OC.

A key element of such a node is its informational structure, which we will call model, as an extension of OC usage. It will reside both in the "subject" and in the "smart object" and will serve as a catalyzer for formation of complex material flows in the node and, at the same time, as an auto-catalyzer for information accumulation. The "vertical" flow of information between model levels (including levels of reflection) shared by "subject" and "object", will be equivalent to reflective control, as described in 4.2. This informational influence is expected to replace, to a large extent, the material forcing exerted by anthropogenic control on natural systems.

Informational interaction between nodes. In such a distributed system, information accumulation should be due mainly to information exchange between nodes, rather than to activity within each separate node. Thus, information (model) becomes distributed - a "collective property" of the entire network. Information flows follow the archetypal pattern of interaction between the central/collective and individual nodes, now in the form of a reflective game described in 4.2. Different nodes are "holders" of different aspects of the model (e.g., subsets of parameters) and/or different scenarios of control/game. Since scenarios are diachronic, the system of nodes should be viewed diachronically, as well: co-existing nodes can hold different time sections of the model, and nodes appearing and disappearing at different times may be parts of a single scenario. Nodes are "co-holders of time" [Mandelstam, 2012] if "time" means not just the calendar counter but, instead, the "meaningful" time.

The simplest regime of information distribution in such a game is the reflective "information equilibrium", an analog of Nash equilibrium described in 4.2 - an extension of the notion of homeostasis. In noospheric situation, this simple regime should be viewed as exceptional: information exchange between reflective levels of partner nodes would make typical the Pareto regime, rather than Nash regime. Nodes will form "coalitions", and spatial or functional structures made of nodes will appear, persist for a while, and yield place to other configurations. This can be viewed as a part of homeostasis or as a form of evolutionary dynamics. The making of each specific configuration of nodes is a noospheric analog of the genetic process of transcription / translation / folding / export. The distributed model is an analog of DNA; the assembly of nodes into a "coalition" is an analog of the assembly of proteins in ribosome; and the value of criterion obtained in a reflective game is an analog of fitness. In GT perspective, individual criteria of partner nodes combine into a collective criterion of their coalition, which is optimized by the nodes' collective strategy. In the same way, one can expect the formation of a collective criterion for the entire noospheric network, notwithstanding the differences in partial criteria of its participants. As all participants are reflective, the entire system is reflective, and the formation of a common criterion amounts to the appearance of a collective "I" in the noosphere, analogous to a collective genetic "I" recognized by the immune system or to a collective behavioral "I" of any organism.

This process includes the appearance of new nodes. Since each node is a holder of information, for new nodes to appear, information has to be first accumulated or generated. Simply speaking, accumulated information comes from geo- and biospheric dynamics external to noospheric system, and generated information is due to the autocatalytic properties in reflective nodes (see 4.2).

Noosphere and geo/biosphere: resonances and "I". In the noospheric context, with its blurred distinction between artificial and natural, the natural criterion of selection by 
reproduction rate should yield place to "artificial selection" by informativity. This is an extension of the tendency to "cephalization" [Teilhard de Chardin, 2008] or "complexification" [Moiseev, 1999] seen throughout the history of Earth. In OC perspective, this is a radical change in the type of control: from changing the states to changing the laws. By "laws", we mean the information flow archetypes acting on all levels: geo-, bio- and noo-. These archetypes support the "information resonances" (alias, "meaning resonances" or "symbolic resonances") between geo-, bio- and noo-.

The resonances are produced by information flows circulating along the noo-bio-geobio-noo sequence, like "vertical" information flows circulating in model layers of OC subject. Each node, which replaces "subject" in the noosphere, incorporates a layering: geo-, bio- and noo-. As described in 4.2, each higher level contains a symbolic representation of the lower level, and the vertical information flow between them induces "projection" and "introjection" - formation of symbolic structures on the way upward and objectification of (changed) symbolic structures on the way downward. By "resonance", we mean the capability of a reflective informational structure to recognize itself in an analogous symbolic structure existing at a different layer, and to react. This is an inherent feature of reflective systems since, for a partner to react properly to its phantom image in another partner, it should be able to recognize itself in this image and distinguish it from the plethora of other images produced by the other partner. "Resonant structures" can be viewed as components of an "I" formed by noosphere. Since noosphere has a diachronic aspect, resonances can be diachronic. E.g., the actualization of a virtual scenario (see Chapter 1 of [Balter \& Faminskaya, 2017]) can be seen as a resonance between this scenario and the future, which "recognizes itself" in this scenario, so that the virtual information structure of this scenario is amplified, and it becomes dominant in the package of virtual scenarios.

The resonance within GBN is a disturbing problem. Noospheric control of biosphere is expected to encroach upon the fundamental biological processes, including ontogenesis and phylogenesis. Production of genetically modified plants is just the beginning. Conversely, the logic of biosphere can be manifested in the noosphere. Some features of the human noos are formed by the Darwinian selection in the biosphere (these are the subject of sociobiology). Humans, seemingly, are no more an object of biological selection, but the logic of speciation may still manifest itself in the noosphere, now involving informational features. Ethnogenesis and formation of cultures, perhaps, herald this future.

However, Darwinian principles carried over literally to the information realm would produce only informational machines - like living creatures seen as biological machines. These can be called golems [Collins \& Pinch, 2002]. The word "technosphere" often used as a substitute for noosphere presents such a partial view. Taken separately from human control, "noosphere" would be a world of golems, and technosphere would have an "I" of a huge "golem" (metaphorically, since golems are by definition incapable of reflection). The transposition of Darwinism to noosphere has to include reflection, e.g., use the hypercyclic view of biogenesis, replacing the biochemical autocatalysis by informational reflective autocatalysis. The reflective formation of a stable "I" would be an analog of the robustness of hypercycle to invasion by competing quasispecies. In noosphere, these stable reflective structures would include the existing technical and the appearing techno-biological production chains - importantly, together with a "conscience" in the form of scientific apparatus supported by these chains and controlling their development. See [Beer, 1994] on the "I" of an enterprise.

This is a picture of co-evolution involving geo-, bio- and noosphere. The question avoided in the Darwinian picture of evolution - "qui bono", i.e., who/what sets the criterion optimized 
by evolution, - returns made even more complicated by reflection. Whereas on non-reflective, biospheric level, we can clearly distinguish between a system and its externally defined criteria, in noosphere, we get instead a plethora of reflective images of external criteriasetting entities. A key to the answer is, maybe, the change of the meaning of "qui bono" in noosphere: now, "bonum" is reinstated in its original meaning as something elevated above purely material interests - first to the realm of information, and then, hopefully, to the realm of meaning. A criteria-setting role slowly but surely acquired by science may be a first step on this way.

\subsection{Science as the distributed controlling model}

Science as informational and social entity. The advent of science is seen by Vernadsky [Vernadsky, 1997] as the main symptom of the noosphere being formed. As everything in the noosphere, science has two sides: material, as a social phenomenon, and informational, as a symbolic representation of reality. These two sides are represented as two components in the criterion optimized by science, so that there is a feedback between them. E.g., social investments into science should be compensated by the control value of acquired information; the information collected by science should reflect the social, including its own role in it. The relationship between intrinsic, esoteric, information-bearing side of science and the external, social side follows the same archetype as the relationship between genetic and phenetic: "informational ecosystem" is connected by feedback with "social ecosystem". So far, science is barely reflective, and this is why scientific and social development is all but unpredictable. Partly, this is due to the general low reflectivity of collective phenomena in contrast to individual ones (see 4.2).

As a social phenomenon, science inherits biospheric mechanisms characteristic for all social phenomena - most importantly, the mechanism of "natural selection" of scientific developments. Additionally, the emerging reflectivity of GBN is expressed as the projection of biospheric laws into the practice of science, which studies them. Reflectivity implies the converse phenomenon, as well: projection of informational structures of science into everyday social life. Evident in the spreading of science-based technological gadgets, this is even more important is the assimilation by "everyman" of scientific logic and scientific thinking techniques, especially those considering the uncertainties and reflecting on one's own possible errors.

Distributed and splintered science. The appearance of science as an institutionalized system of information handling is inevitable in a distributed noospheric information structure where the exchange of information is the major process. Institutionalization opens the way to systematic handling of stochasticity and uncertainty (which are evident, e.g., in relativity, quantum theory, incompleteness theory), which is the reason for using OC and GT as a language here. Without institutionalization, accounting for uncertainty in communication between individuals quickly dies out, and only "true" or "false" remains as a final judgment, while institutional informational structure supports more refinement.

The distributed nature of science facilitates its branching - an analog of speciation. As in biological evolution, branches tend to communicate and establish "informational ecosystems" without losing their own identity. The holistic image of reality characteristic to pre-scientific societies splits into a hierarchy of reflective images in the multiple mirrors of various sciences. (The image of noosphere in this paper is an example.) As a result, the sharp borders of reality are blurred and intertwined with fragmented pieces of model; the unified, objective view of reality - the goal of natural science for at least four centuries - 
gives way to inter-subjective views, each shared by a coalition of researchers. As shown in 4.2 , this is a natural development in a game between reflective partners - here, nodes in the scientific network.

Nevertheless, scientific language finds its way into everyday life - at least in the form of "scientific memes" like "selfish gene", "intelligent design" etc. They need not be comprehensible, to be effective in catalyzing the formation of noosphere. The material support of the forming noosphere is a social phenomenon and it depends on anchoring the informational part of the noosphere in social life. It is no accident that the first glimpses of noosphere were caught popular images in science fiction. Social coherence is expected to drive the integration between different branches of science - at least in the form of a common language or of a "translator". Mathematics is the current inter-scientific "Esperanto". Its limitations are well known but it is not clear what could be an alternative translator between different sciences. In this paper, OC principles are tested for this role.

Science as a part of culture. A fuller view of science in the noosphere sees it as a part of culture - of a wider information structure. The role of culture is double: it accumulates information as science does, but it provides meaning, an answer to the "what for?" question that science, focused on "why" and "how", deliberately avoids. OC has some tools for "what for": criterion, goal, goal-oriented scenarios, causality inverted in time. Thus, one can envision the integration of science and other, sense-oriented components of culture: art, religion, etc. Historically, science was born from this unity, and Newton or Descartes viewed it as a quest for meaning. For them, science was like deciphering the musical score - not for the mere joy of it but for a live performance (in OC language, not just for constructing a model but for applying it in control). So far, science looks more like a reader of a symbolist poem who is excruciatingly trying to put together the elements of the plot and is still far from seeing the beauty of it. Every reader (or, in the musical metaphor, every performer) forms an individual interpretation of the text/music. While the subsequent exchange of impressions creates a kind of collective perception, it is inevitably flat - the depth remains at the individual level. So far, science is not capable of forming such complex individual-collective structures, but noosphere will need it.

\subsection{Formal and natural languages in the noosphere}

Natural languages in the noosphere as extensions of the genetic language. Science is centered on formal languages (especially, mathematics). However, at least as great a role in the noosphere is played by natural languages, which are among the first noospheric phenomena. They are accumulators of relevant (sensible) information extending the function of DNA in biosphere to noospheric context. That is why DNA is often compared to text and genetic code to language. As in genetics, the functioning of natural languages involves transitions between individual and collective in the process of natural selection. As shown in Chapter 3 of [Balter \& Faminskaya, 2017], genetic coding of environmental information includes "symbolic" transformations; languages are the main carriers of symbolic meanings, as well. Symbolization is a multistage process, and, thus, information accumulated in languages has a multi-layer structure. This includes the reflective mapping between different layers; in fact, language is the main instrument of reflection for humans.

Language exists in two main forms: as a spoken, informal phenomenon and as a set of texts, including the self-description of language. In the genetic analogy, texts can be mapped either to the genotype accumulating information, from which proceed the spoken utterances, or, conversely, to the phenetic objectification of an informal spoken "genotype". These two 
approaches amount to a half-turn of the reference frame for the genetic $\longleftrightarrow$ phenetic cycle. Although text per se has no intrinsic dynamics, it acquires dynamics in the reflective dialog with the reader who projects reflective images into the text and introjects the result of their interaction. Since this is how model works in the OC cycle, texts can be regarded as models existing in the noosphere and driving its dynamics. In contrast to many other types of models (programs, etc.), texts, in addition to information, carry meaning.

If information is considered as the syntactic level of text, meaning is the semantic level. We use these terms in a somewhat specific way adapted to OC context: syntactic level carries answers to "what" and "how", and semantic level, to "why" and "what for". Language products that strive at univalent interpretation - e.g., technical texts - usually do not carry answers to the latter two questions. If a text carries meaning at all, it is usually a packet of several possible ("virtual") meanings. This is an analog of the polysemantic ambiguity of DNA. In the integrated view of geo-, bio- and noosphere, due to interaction with noosphere, many geospheric and biospheric phenomena can acquire the quality of "texts" and carry meaning (of course, manifested only through interaction with noosphere). The meaningful role that landscapes, beauty of plants and animals etc. play in human perception and their abundant mapping to texts are a testimony to their "textual potential".

Evolution of language. Historically, the "language universe" is expanding like the physical universe or the "universe" of life forms. It has been often remarked that evolution of languages is, in many respects, similar to the evolution of life. It includes homeostasis, bottlenecks, crossover, speciation, reproductive isolation, etc. In the process, while a linguistic element (first of all, word) can remain syntactically more or less the same, its semantic spectrum (the range of virtual meanings) can significantly widen, narrow, or shift. Widening is an analog of accumulating the neutral genetic variations, and it is an important evolutionary tool when a language gets out of homeostasis. Narrowing happens when, e.g., a word is constrained to a specific, technical usage as a part of a "terminological" word set. Then, a part of language information stabilizes to a homeostatic state and becomes immune to the variations of the spoken language. This is how scientific terminology is created. In addition to these "phylogenetic" features of languages, there are "ontogenetic" features, which define the understanding and usage of language in individual development. When individuals with different personal understanding/usage of language become partners in a dialog, they encounter the problem of reference frame reconciliation described in 4.1. In OC terms, each partner uses language as a "transformer" both in understanding and in controlling the other partner, so that reconciliation is obtained when the information flow in OC cycle, including the observation and control information, stabilizes. In GT terms, reconciliation is a part of searching a common, cooperative Pareto optimum. As a result, the semantics of the words used changes for all partners, and if this type of dialog is repeated often, the change percolates from the individual level to the collective level, driving the evolution of language.

Variety of noospheric languages. In addition to spoken/written languages, the emerging noosphere includes many other information structures that function as "languages": rituals, standard narratives (historical, political...) etc. These social structures arise naturally, as do other tools for accumulating, filtering and assimilating information. These structures function even better than spoken/written languages in filtering the meaningful information from the irrelevant, which becomes particularly important with the current informational deluge. As any meaning handler, they address the deep, reflective layers of the human psyche and thus generate complex informational phenomena. One of them is the paradoxical type of logical inference described in 4.2 - "illogical logic". Another is the polysemantic meaning - a kind 
of uncertainty relation. Yet another is the "mirage" effect: reflective images, which have no correspondence in reality. Noosphere is not a purely rational entity (after all, it involves the human subconscious), and thus it has to assimilate the "languages of irrational". A prototype of such assimilation is art, and it is expected to play an important integrative role in the noosphere, analogous to the role of science for rational.

Formal and informal languages in the noosphere. To transcend the gap between terminology used for geosphere, biosphere, and noosphere, we need a language meaningful at all three levels. OC language used in this paper is a candidate but not the only one possible. It is a formal, technical language that has to be supplemented by other types of language like those mentioned above. Noosphere will include, perhaps, the symbolical information structures analogous to such linguistic constructs as comparisons, metaphors, etc. They may be integrated into OC as interpreters of the meaning of formal model-based "texts", which adequately transmit virtuality, uncertainty, ambiguity present in these to human participants of OC. The first glimpse of the potential role on non-formal languages in the noosphere is the fact that they obtained the status of the main tool for information search on Internet.

Speaking scientifically about noosphere calls for an extension of scientific language, which should include built-in means of expressing the uncertainty of statements, their reflectivity, inherent Gödel-type incompleteness etc. Current scientific language excels in its unambiguity, but this is attained at the cost of expressive power. These two poles of language capabilities are analogs of the exact information conservation vs. adaptive open-endedness in genetics. Thus, in addition to the exact logical inference, noosphere should use "poetical inference" [Mandelstam, 2012] as the transcendence of the limits of unambiguously provable. Often the usual scientific logic is called linear; then, its noospheric complement should be circular, or dialogic. Applying the $\mathrm{OC}$ notions to such circulating logical inference, one expects the reproduction in logic of a familiar adaptive OC feature: drift of reference frames - of axioms and meanings of basic notions - along the inference cycle. On the other hand, inferences starting from different reference frames might converge in repeated "logical OC" cycles to some attractor. Both phenomena can be observed in the historical development of science, but in noosphere, they are expected to happen "online".

The unambiguous, mono-semantic pole of the spectrum of languages is best represented by the programming languages - even better than by the language of science. They are already indispensable in OC and, obviously, they will keep their role in noosphere. Although artificial, they inherit the genetic approach to control as programming, as in DNA. They also inherit from natural languages the capability of reflection expressed in the form of recurrent procedures (although without symbolic mapping between layers of different type). These features make possible, in principle, the intrusion of programming into human thinking and into genetic language. These disquieting but, perhaps, inevitable features of the noosphere are discussed below.

\subsection{City as an emerging integrated noospheric structure}

Historical heritage of city. Many pieces of noosphere are already in existence but very few form complete integrated structures - "noospheric microcosms". A notable exception is city. Like much in noosphere, it inherits several cardinal biospheric structures: separation of internal environment from external (historically, by walls similar to cellular membranes); development as a node in the network of flows (historically, flows of trade, nowadays mostly information flows, including financial information). We focus on OC perspective: control cycles operating in city. 
Early stages of most modern cities go back to Middle Ages when cities were aiming at homeostasis rather than evolutionary development. In modern age, cities are fast evolving units. This does not exclude the existence of important inertial, inherited structures, especially the spatial patterns, like many genetic and phenetic features extant in evolving species. The reason is the same: balance between information storage and adaptiveness. Spatial patterns serve as a link between city and its geospheric environment, so that they cannot change fast. Slowly changing self-organized spatial structures are, perhaps, the most evident "evolutionary observable" in cities. On the other hand, the homeostasis of a city is most visible in its daily ebb and flow. Thus, we have to distinguish at least two time scales in the city: fast and slow, as we did for general adaptive OC systems in Chapter 1 of [Balter \& Faminskaya, 2017].

Control cycles in city. City participates, of course, in the natural geospheric cycles of heat, humidity, etc. However, an important function of the "wall" separating the internal and external environments consists in screening the city from these cycles. Perhaps, the most obvious example is the heating system. This and many other control cycles running in "fast" time are engaged in keeping the homeostasis, which consists of intertwined natural and technical cycles. Historically, many of these cycles: heating, water supply, food supply, etc. have been running in "automatic"" regime as feedback responses to the driving changes of environment. Recently, these cycles more and more rely on information processing, forecasts and scenarios (weather forecast is an obvious example), which is a symptom of noospheric development. In the noospheric context, we are mostly interested in the information flows in cities. These should be more pronounced in control cycles supporting evolution, rather than homeostasis.

One of the most visible control cycles of this kind is that aimed at solving the problem of transport structure (in contrast to the everyday control of transport jams.) The tools it uses are the development of road networks, territorial specialization, establishing the rules for migration, etc. This control cycle and its likes use the most refined modeling instruments in city practice. They include active sounding in the form of "try and learn" experiments. However, so far, the full power of OC is not used here. A perspective direction is the application of reflective control, taking into account the fact that city controllers are parts of the city and are driven both by common and by private interests. Another manifestation of reflectivity is the fact that publication of control plans induces an immediate reaction from the city and, thus, changes the situation for which the plans were optimized.

Perhaps the most important control information flow is that of money. As described in Chapter 1 of [Balter \& Faminskaya, 2017], criteria optimized in control contain both the material part and the informational part, so that in OC money flow is convertible to information flow and back. In this perspective, the historical extension from city as a monetary hub to city as an information hub is natural.

There are so many stakeholders in any city that what we called OC includes a considerable GT component. The appropriate framework for combining GT and OC is the distributed control network where the co-controlling nodes play out their respective gains between them. Partly, these nodes are territorial, partly functional. Therefore, a possible simplifying approach to city control considers its information flow as composed of interacting but quasiindependent threads of different functional subsystems: public utility services, financial system, transport system etc. Each thread can be construed as a node representing a coalition of partners in the "city game". In everyday homeostatic regime, these partners normally stick to the established Nash equilibrium balancing their respective gains and costs. However, in evolutionary regime, when radically different scenarios of the future development are 
generated, these partners would engage in information exchange and bargain in search of a new Pareto equilibrium (which, in a later temporary homeostasis, will again become a Nash equilibrium).

Global city - model of noosphere. Models discussed in [Balter \& Faminskaya, 2017] were naturally associated with equations or computer algorithms. However, city can be seen as a "living model" of noosphere anticipating its features and preparing for being actually used as a noospheric information structure. City as a functioning model resident in global noosphere has to be a globally distributed entity. Its regulatory role with respect to geospheric and biospheric processes makes it an extension of Gaia (see Chapters 2, 3 of [Balter \& Faminskaya, 2017]). As described there, hitting the spatial boundaries and developing the global "sphericity" is a necessary condition for developing the "vertical" information flows that emerge from the "horizontal"" ones.

The current process of globalization is mostly viewed through the market lens, and its models are predominantly those assuming the rational market agents. It is well known that this view, while amenable to mathematical modeling, is biased. If we consider city as a model (including mathematics as one of its reflective layers but not reducing model to it), the picture becomes more adequate. Monetary flows and market as an "optimizer" form just one functional subsystem, sometimes cooperating and sometimes competing with other subsystems. Obviously, in the emerging noosphere, economic and political globalization is only a part of several cooperating and competing information flows. As mentioned above, there is a kind of convertibility between economic and informational values, so that growing informational flows in the noosphere are creating a market of information, which may easily overwhelm the traditional one. (The crisis associated with financial derivatives may be a symptom of this process.)

A considerable part of global information market will be formed, probably, by the model scenarios of global and local evolution. Production and usage of such scenarios is quickly becoming an element of culture, beginning with the famous global dynamics [Forrester, 1973]. However, the noospheric "scenario culture" will be reflective: will include the backfiring of scenario on the reality on which it is based. This reflective effect is currently best seen in the reaction of city population to the publication of municipal plans, information on threats, weather forecasts etc. This is bound to become a global phenomenon. In this respect, as in others, noosphere will be a global extension of what we see in city, with information channels as a skeletal infrastructure, similar to piping or sewage.

Individual and collective in city. As mentioned above, the logic of relationships between individual and collective is a key issue in distributed OC, as well as in collective GT. This problem is inherited by the noosphere from the biosphere where there are many types of individual-collective equilibria in animal and plant collectives. (This variety exists at the phenotypic level, while at the genotypic level the only relationship we know is universal: that between species or population and its individual representative.) Early human collectives inherited a subset of these biospheric solutions, seemingly without inventing anything radically new (at least as far as we can judge from the present isolated aboriginal populations). However, the cultural information accumulation made possible by human speech had produced a radical restructuring of individual-collective relationship and thus, the city. Characteristically, simultaneously with city appears writing as a new information accumulation tool, which shows that the root of change is an informational process. Individualcollective relationship in early cities was based, as testified by the written sources, on force similar to certain animal collectives. However, the inexorable information processes have 
driven city through many unique historical forms to what it is now: a reflective distributed information accumulator and controller allowing a wide range of individual choices. It is this individual freedom and inventiveness that is the driver of evolutionary potential of city. Virtual information created individually is amplified and actualized by interaction with the collective and makes the previous collective scenarios obsolete. This inexhaustible newness is shown especially vividly by the last century or two of city development elevating to a new level what has been shown by the billion-year biological evolution. This is, perhaps, the key to understanding the role of individual in the noosphere.

\subsection{Person and noosphere: rational, irrational, and self-controlled}

Role of person in noosphere. In [Balter \& Faminskaya, 2017] and in 4.4 and 4.6, it was shown that the collective success of distributed OC implies active interaction between individual nodes according to GT. Among the nodes, there are spatial conglomerates and functional coalitions, but the most important type of node is human person. A symptom of this is the transfer of "efforts" of natural selection from collectives (species, populations) to individual humans, so that information is accumulated in cultural rather than genetic domain. This phenomenon is active already for many millennia.

The unique role of person is that of information generator and meaning carrier. Therefore, probably, personal activity in noosphere will look more like a game than like current work or strife where person is mostly workforce and consumer rolled into one. However, this description of personal activity in the noosphere is limited by the limits of OC and GT where controllers and players are rational gain-seekers and consumers. Essentially, there is no place in OC or GT for a non-repeatable entity called "personality". Therefore, attempts to look at noosphere through purely scientific OC or GT lens, like in this paper, caricature noosphere as a community of reflective automata. Attempts to correct this bias inevitably introduce a humanitarian, subjective component into the picture drawn with technical, objective tools. Thus, we have to recognize the unavoidable personal, subjective element in noospheric science. How can it combine with the necessarily collective character of science as the model driver of the noosphere? Perhaps, the answer lies in the Russian religious term "sobornost", which, applied to scientists, looks at them as a kind of religious community linked by irrational, in addition to rational, links. This, of course, does not exclude the complementary views of science as a community of seekers of objective truth or as a societal group; however, the early scientific communities (e.g. Pythagoreans) show symptoms of this undefinable feature. A glimpse of this type of link appeared in the discussion of Prisoners' Dilemma in 4.1 where it was construed as force let us call it trust - which was driving the partners to cooperative action. This force has an ethical nature, like sobornost, which is essentially an ethical notion, so that, in discussing this issue, we inevitably pass into the realm of irrational.

Ethical issues in noosphere. Ethical conscience is an attribute of individual psyche. However, if we understand noosphere, with Florensky [Florensky, 2000], as a projection of the features of microcosm to macrocosm, noosphere looks as an ethical entity. This side of noosphere was emphasized also by Moiseev [Moiseev, 1999]. Of course, human collectives known heretofore are not reflective entities so one cannot speak of their specific ethics, only of that inherited from their component individuals. Noosphere, apparently, will be different. Its ethics, probably, will be something different from individual ethics, so that we should cautiously speak of an "ethical resonance" between noosphere and its constituent individuals. Due to this resonance, the noosphere can serve as a harmonizer between different 
personalities or between different reflective levels in a single person - as the beauty of nature sometimes does (in contrast to its rationalization as "environment").

Differences between reflective levels in a single human psyche can be as great as differences between persons. In GT terms, there can be different players in a single human. This creates obvious ethical problems for this person, so vividly described by Dostoyevsky. One of the lessons drawn from this microcosmic experience is that inner ethical conflicts (even unconscious) constrain the accuracy and depth of reflection, thus leading to selfdelusions or to deliberate coarsening of the worldview (in OC terms, to flattening of the model). These lessons are applicable to the macrocosmic noosphere, as well.

Ethical self-control of the noosphere can be a necessary component of its homeostasis. Without it, one would expect the susceptibility of noosphere to the intrusion of primitive informational "memes" (see 4.2), e.g., to totalitarian propaganda and the like. Historical experience shows that such intrusions are almost inevitable in human collectives based on power interactions and on material exchanges - but noosphere will be based on exchange of information and meaning. If one looks at ethics as one of the symbolic information layers (which is a simplification), noosphere will intertwine it with the material layer like halves of a symbol (in the etymological sense of the word). Evolution researchers have shown that a rough prototype of ethics could have appeared as a result of natural selection even in groups of very primitive real or simulated agents. Such phenomena can be viewed as glimpses of future noosphere seen in biospheric context. However, due to ineradicable limitations of "hard science", this proto-ethics or quasi-ethics is the ethics emerging from rational behavior, while Prisoners' dilemma shows a situation where cooperation is, conversely, a rational behavior growing from pre-existing ethics. Still, the "ethical resonance" of the individual proto-ethical breakthroughs with the collective ethics can amplify them to the full ethical scale. This feedback between microcosm and macrocosm may show how the noospheric ethics can arise. Regrettably, as long as noosphere is still in its infancy, this amplification of the individual by the collective usually worked in the opposite direction and enhanced the anti-ethical ideas and behaviors.

The main component of the keystone idea of noosphere by Fedorov [Fedorov, 1990] was ethical: the restoration of the dead ancestors. This idea should not be understood in an entirely materialistic way but as restoration of the uncorrupted conception of human individual as a microcosm (including the contents transmitted along the ancestral line) linked to Humans as a macrocosm.

Identity of "I" in the noosphere. Individual identity, as understood through OC/GT lens, is the product of interacting virtual variants of self-understanding, and results in behavior that feeds back on self-understanding. The problem of "I" is almost unexplored in hard science, even in reflective GT where it is deliberately avoided by dealing only with the reflective images of others. What was called above "personality" can be formally understood as a result of fusion between reflective phantom images of oneself. Since identity is an essentially reflective phenomenon, interaction with other reflective partners can change one's identity. Of course, this is not an easy reformation - it is one of the "catastrophes", as defined in [Balter \& Faminskaya, 2017]. If one is immersed in an informationally powerful reflective system, such as noosphere, losing one's identity is a real possibility. The solution to this issue may lie along the same line as the solution of the analogous biospheric issue: preservation of biochemical identity of barely formed proto-living creatures. In [Eigen \& Schuster, 1979], it was shown that hypercyclic organization in a circle of auto-catalyzers is a possible solution (see Chapter 3 of [Balter \& Faminskaya, 2017]). One can envision an analogous "vertical 
information hypercycle" running between reflective levels of a person. Alternatively, one can envision a hypercycle made of several cooperating identities, which support themselves by participating in a "collective identity". However, presently, we can hardly see the full scale of this problem, which can be called "ecology of psyche", in analogy to "computation ecology" (see 4.3). Anyway, the "ontogenesis" of a personality in the noosphere will have to become a goal-oriented activity, in contrast to what it is now, including education and self-education. Due to the limits on conscious information processing capability of a single person, it can be expected that high levels of reflection developed in this process would pass into the realm on unconscious, e.g., acquire the form of emotions. Thus, some analog of "Education sentimentale" should appear.

As noted above, noosphere can develop partial identities or even an identity of the noosphere as a whole. Such events are abundantly described in science fiction, usually as something frightening. Indeed, such processes are completely unpredictable, thus activating the fear of unknown.

\section{[Dd References}

Axelrod, Robert. The Complexity of Cooperation. Princeton University Press, 1997.

Balter, Boris, and Marina Faminskaya. Noosphere as Optimal Control. Part 1. Control Theory,

Geosphere and Biosphere. Philosophy and Cosmology, Vol. 19. Kyiv: ISPC, 2017: 12-32.

Beer, Stafford. The Heart of Enterprise. Wiley, London, 1994.

Collins, Harry, and Trevor Pinch. The Golem at Large: What You Should Know About Technology. Cambridge University Press, 2002.

Dawkins, Richard. The Selfish Gene. Oxford University Press, 2006.

Eigen, Manfred, and Peter Schuster. The Hypercycle: A Principle of Natural Self-Organization. Springer, 1979.

Fedorov, Nikolai. What Was Man Created for? The Philosophy of the Common Task: Selected Works. London: Honeyglen Publishing, 1990.

Florensky, Pavel. Letter to V.I. Vernadsky, 1929. In “Collected Works in 4 Volumes”. Moscow: Nauka, 2000 (in Russian).

Forrester, Jay. World Dynamics. Cambridge, Mass.: Wright-Allen Press, Inc., 1973.

Hofstadter, Douglas. Gödel, Escher, Bach: An Eternal Golden Braid. Basic Books, 1999.

Huberman, Bernardo. The Laws of the Web. Patterns in the Ecology of Information. The MIT Press, Cambridge, Mass., 2001.

Kant, Immanuel. Critique of Practical Reason. Indianapolis/Cambridge: Hackett Publishing Company, 2002.

Mandelstam, Osip. Conversation about Dante. In Osip Mandelstam. Selected Essays. Austin: University of Texas Press, 2012.

Moiseev, Nikita. Reflection on the noosphere - humanism in our time. In The Biosphere and Noosphere Reader-Global Environment, Society and Change. London, New York: Routledge, 1999.

Novikov, Dmitry, and Alexander Chkhartishvili. Reflexion and Control: Mathematical Models. CRC Press, 2014.

Teilhard de Chardin, Pierre. The Phenomenon of Man. New York: Harper Perennial Modern Classics, 2008.

Vernadsky, Vladimir. Scientific Thought as a Planetary Phenomenon. Moscow: Nongovernmental Ecological V.I. Vernadsky Foundation, 1997. 